\title{
Fundus Image Enhancement by Using Maximum Entropy and Perona-Malik Diffusion Filter
}

\author{
Syaiful Anam, Zuraidah Fitriah, Nur Shofianah and Ratno Bagus Edy Wibowo \\ Mathematics Department, Brawijaya University, Indonesia
}

\begin{abstract}
Diabetic retinopathy is one of the common complications of diabetic diseases. Diabetic retinopathy causes leakage of blood vessels or hemorrhage, and distorting vision. The blood vessels of diabetic retinopathy patient may swell and leak fluid. The abnormal blood vessels may grow on surface of retina. Diabetic retinopathy causes changes in the eye, the disease may affect the vision. Early diagnosis of diabetic retinopathy is very important to prevent blindness. Ophthalmologists use the retina image (fundus image) of the patient to diagnose diabetic retinopathy. The exudates in fundus image are primary signs of diabetic retinopathy. However, the fundus image often contains noise and illumination of uneven fundus image, it is very hard to detect the exudates in some images. For this reason, a method is needed to enhance the fundus image for segmenting the exudates in fundus in the next process. The maximum entropy method has been applied succesffuly for image image segmentation. Perona-Malik diffusion filter is one of the alternative filters that can reduce noise as well as effectively maintain the edge of the image This paper proposes the use of maximum entropy and Perona-Malik diffusion filter to improve the quality of the fundus image. The quality of a good fundus image will facilitate the ophthalmologists in detecting exudates in the fundus image.
\end{abstract}

Keywords: fundus image, maximum entropy, Perona-Malik diffusion filter, image enhancement.

\section{Introduction}

The diabetic vascular complications are classically classified into microvascular and macrovascular which would result in heart disease, renal problems and retinopathy. Diabetic retinopathy is the most common microvascular complication of diabetes mellitus. Diabetic retinopathy is the leading cause of blindness in the working age population in the western world [1]. This disease begins with a narrowing of blood vessels in the eye. This narrowing of the blood vessels may cause leakage of blood vessels or haemorrhage (bleeding) and the accumulation of fluid and fatty material in the retina. These conditions cause the blurred vision. If these conditions is not treated then it can cause severe vision damage as well as blindness. The risk of diabetic retinopathy can be reduced by early detection, controlling of sugar, blood pressure, and lipid appropriately [2].

A fundamental analytic procedure in ophthalmology for diagnosis of diabetic retinopathy involving structural and functional transformations in the vasculature is the analysis and interpretation of the retinal images. Retinal images are known as called fundus images [3]. The vital manifestations of diabetic retinopathy and retinopathy of prematurity and cardiovascular risk are the changes in retinal vasculature, such as haemorrhages, angiogenesis, and increase in vessel tortuosity, blockages and arteriolar-venular diameter ratios [4]. Changes in blood vessel diameter, microaneurysms, lipid and protein deposits also known as hard exudates and cotton wool spots depending on the features, haemorrhages and new vessel growth are all characteristics of diabetic retinopathy [5]. Ophthalmologists often use exudate on fundus image for diagnosis of diabetic retinopathy.

The exudate detection is an extremely exhausting and time consuming task for ophthalmologists because the retinal image taken by fundus camera often contains noise and has uneven lighting. In addition, the details of retinal fundus images such as small blood vessels, microaneurism, and exudate often have low contrast. To improve exudates visualization, noise reduction and an enhancement of fundus image are very important tasks in the case of pre-processing of fundus image. For this reason, a method for enhancement of fundus image for detecting exudates is strongly required. As the representative conventional noise reduction methods, the median 
filters [6], morphology analysis [7], bilateral filters [8] are well-known, but in the same time the exudates boundary also becomes dull unexpectedly by applying those methods.

Above all those methods, Perona-Malik Diffusion (PMD) filter [9] is known as an effective edge-preserved smoothing method. In [10] and [11], the normal PMD filter is used to reduce the speckle noise. However, when the normal PMD filter is applied to fundus image, the exudate boundary cannot be preserved on several areas. The speed function of diffusion are very important factors to enhance the image edges and to reduce the noise. In the normal PMD filter used in $[10,11]$, the speed function of diffusion is controlled by gradient of image. The value of speed function is the opposite of the value of image gradient. The value of image gradient is high in boundary area and low in other areas. However, some boundary of exudates is unclear, if the information of image gradient is used for diffusion proccess, the result is not good. Maximum entropy modelling has been successfully applied to imauccge processing, computer vision, spatial physics, natural language processing and many other fields. Maximum entropy also has been successfully for edge detection in images [12]. For this reason, we propose improving exudates visualization by enhancement fundus image using maximum entropy and Perona-Malik diffusion filter.

\section{Methodology}

Research method used in this research is literature study and experimental. Data is taken from secondary data. In the first phase conducted in this study to examine some theories that will be used in the discussion on this paper such as diabetic retinopathy and fundus image, maximum entropy and Perona-Malik diffusion filter.

\subsection{Diabetic Retinopathy and Fundus Image}

Diabetic retinopathy is a microvascular complication caused by diabetes mellitus. Diabetic retinopathy can lead blindness. In early stages of diabetic retinopathy typically there are no visible signs but the number and severity of abnormalities increase during the time. Diabetic retinopathy typically starts with small changes in

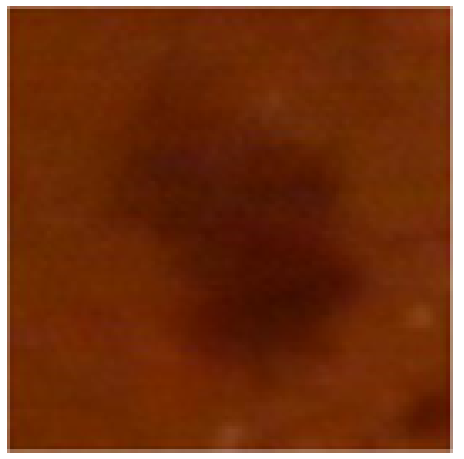

(a)

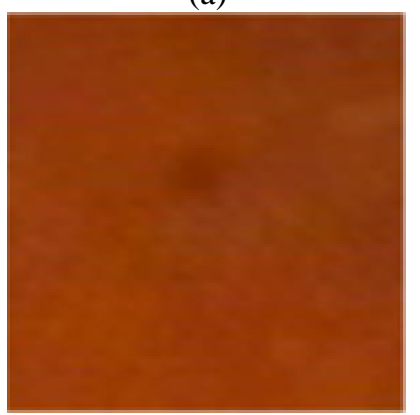

(d)

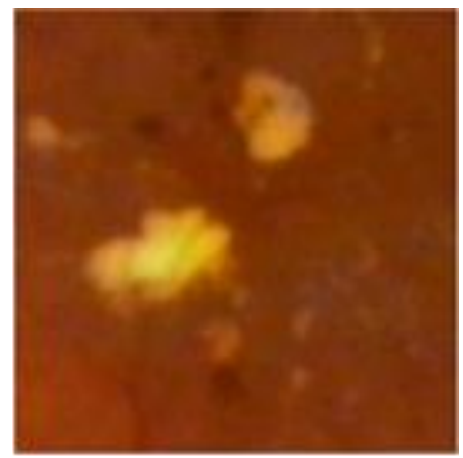

(b)

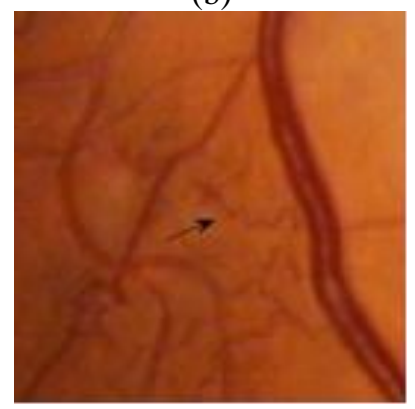

(e)

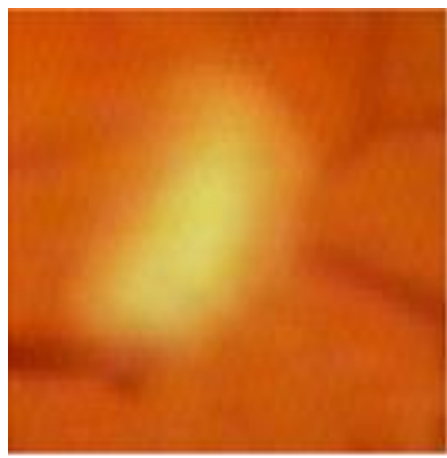

(c)

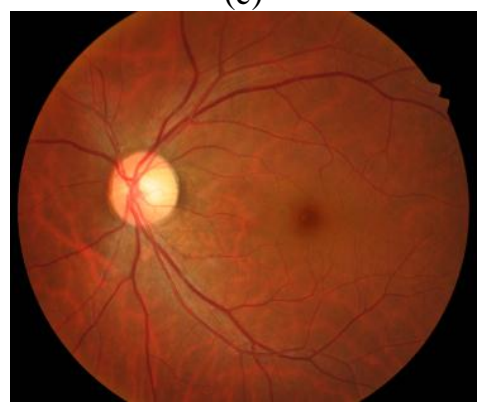

(f)

Fig. 1. Abnormal findings on fundus images caused by diabetic retinopathy. (a) Hemorrhage, (b) Hard exudate, (c) Soft exudate, (d) Microaneurim, (e) Neovas-cularization, (f) Fundus image. 
retinal capillaries. The first detectable abnormality is microaneurism which causes enlargement of retinal capillary enlargement. The ruptured microaneurism can cause hemorrhages to be seen in Fig. 1.(a). After that, it may appear hard exudate as shown in Fig. 1.(b). Hard exudates are lipid formations leaking from weakened blood vessels. Along with the severity of retinopathy disease, the blood vessels may become inhibited causing microinfarct in the retina called soft exudates as shown in Fig. 1.(c). The lack of oxygen caused by microinfarct causes the development of new fragile vessels (neovascularization), as shown in Fig. 1.(d). This phenomenon can cause a sudden loss of vision. The diagnosis of diabetic retinopathy using fundus image is necessary because the disease is progressive, the example of the fundus image can be seen in Fig. 1.(f).

\subsection{Maximum Entropy}

Maximum entropy algorithm is a segmentation method which based on the histogram of the image. The entropy of the image is defined as in (1), where $x$ is the gray level of the image (from 0 to $\mathrm{L}$ ) and $p(x)$ is the probability of the pixel which gray level is $x$ in the image.

$$
H=-\sum_{x=1}^{L} p(x) \log p(x)
$$

The segmentation threshold $t$ will divide the image into two parts: $\operatorname{target} A$ and background $B$. Assuming that the gray values which less than the threshold of the pixels belong to the target $A$, the gray values which greater than or equal to the threshold of the pixels belong to the background $B$, then the probability of pixels in the image are related to target A and background B respectively defined as in (2) and (3).

$$
\begin{gathered}
p a=-\sum_{i=0}^{t-1} p_{i} \\
p a=-\sum_{i=t}^{255} p_{i}
\end{gathered}
$$

The entropy of the target region and the background region are respectively defined as in (4) and (5).

$$
\begin{gathered}
H_{a}(t)=-\sum_{i=0}^{t-1}\left(\frac{p_{i}}{p a}\right) \log \left(\frac{p_{i}}{p a}\right) \\
H_{b}(t)=-\sum_{t}^{255}\left(\frac{p_{i}}{p b}\right) \log \left(\frac{p_{i}}{p b}\right)
\end{gathered}
$$

The entropy of the whole image is defined as in (6).

$$
H(t)=H_{a}(t)+H_{b}(t)
$$

The greater the value of function $H(t)$, the more information of the target and background represent, the more accurate the target and the background area. The global optimal threshold (let it be $t^{*}$ ) is the value which make $H(t)$ take the maximum value, that is, $t^{*}$ can be expressed as in (7) [12].

$$
t^{*}=\arg H(t)
$$

\subsection{Perona-Malik Diffusion (PMD) filter}

An anisotropic diffusion filter, is known as Perona-Malik Diffusion (PMD) filter, is proposed by Perona and Malik have proposed. It is used to filter noise and preserve the edges of an image. The basic idea of the PMD process is to get an increasingly smoothed image $u(x, y, t)$ from an original image $u_{0}(x, y)$, indexed by diffusion parameter $t$. This process can be interpreted as an image convolution by a Gaussian kernel $G(x, y, t)$ with an increasing width as in (8).

$$
I(x, y, t)=I_{0}(x, y) * G(x, y, t)
$$


The PMD filter equation is defined by (9), where $c(x, y, t)=g(\|\nabla I(x, y, t)\|)$ is a diffusion coefficient. $\nabla I$ denotes a gradient of an image.

$$
\begin{aligned}
I_{t}=\frac{\partial I}{\partial t} & =\operatorname{div}(c(x, y, t) \nabla I) \\
& =c(x, y, t) \Delta I+\nabla c(x, y, t) \nabla I
\end{aligned}
$$

$g(\cdot)$ refers to an edge stopping function, which is a decreasing function of the gradient of image, which is defined by (10).

$$
g(\nabla I)=\frac{1}{1+\left(\frac{\nabla I}{K}\right)^{2}}
$$

$K$ is a parameter which controls the strength of diffusion. $g(\cdot)$ takes large values at the regions where the intensity gradients are low. On the contrary, it takes low values at the regions where the intensity gradients are high.

The discrete version of PMD process is defined as (11), where $s=(x, y)$ and $p$ are the coordinates of the pixel of concern and its neighboring pixels, respectively. $I_{s}^{(n)}$ is an intensity at $s$ with an iteration count $n$. $\phi_{s}$ represents the four neighboring pixels in North, West, South and East diffusion directions. $\left|\phi_{s}\right|$ is the number of pixels in the neighborhood area. $\lambda$ is a parameter. The initial condition is given by $I(x, y, 0)=I_{0}(x, y)[9]$.

$$
I_{s}^{(n+1)}=I_{s}^{(n)}+\frac{\lambda}{\left|\phi_{s}\right|} \sum g\left(\nabla I_{s, p}^{(n)}\right) I_{s, p}^{(n)}
$$

\section{Experiment Result and Discussion}

In this paper proposes a method for enhancement of exudate visualization on fundus image using PeronaMalik diffusion and maximum entropy. The data in this research is a fundus image as shown in Fig. 2. The flowchart of the proposed method can be seen in Fig. 3. It can be seen that the first step is to input the the fundus image. Furthermore, the image in the RGB format is converted to CIE L*a*b format and taken b component from CIE $\mathrm{L}^{*} \mathrm{a}^{*} \mathrm{~b}$ image. The final step is to enhance fundus image by Perona-Malik diffusion and maximum entropy. The stages in detail will be discussed in the subsection.

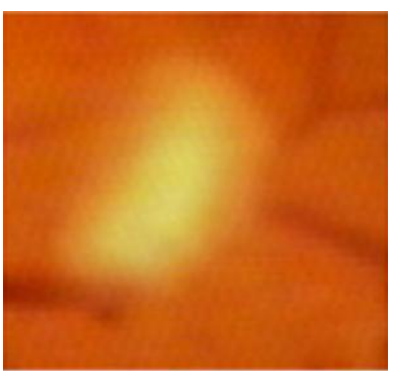

(a)

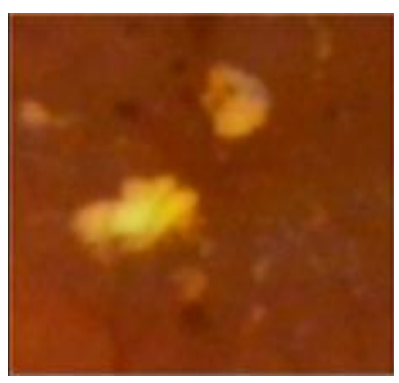

(b)

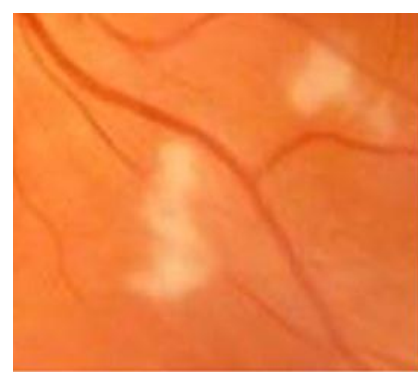

(c)

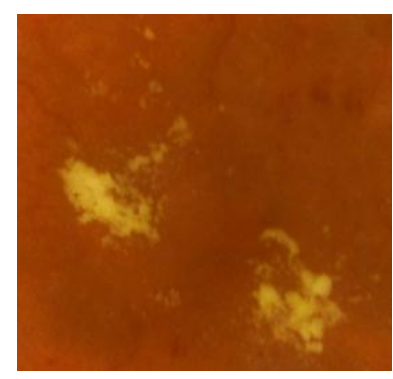

(d)

Fig. 2. Fundus image data used for the experiment. (a). Image $1^{\text {st }}$. (b) Image $2^{\text {nd }}$. (c) Image $3^{\text {th }}$. (d) Image $4^{\text {th }}$. 


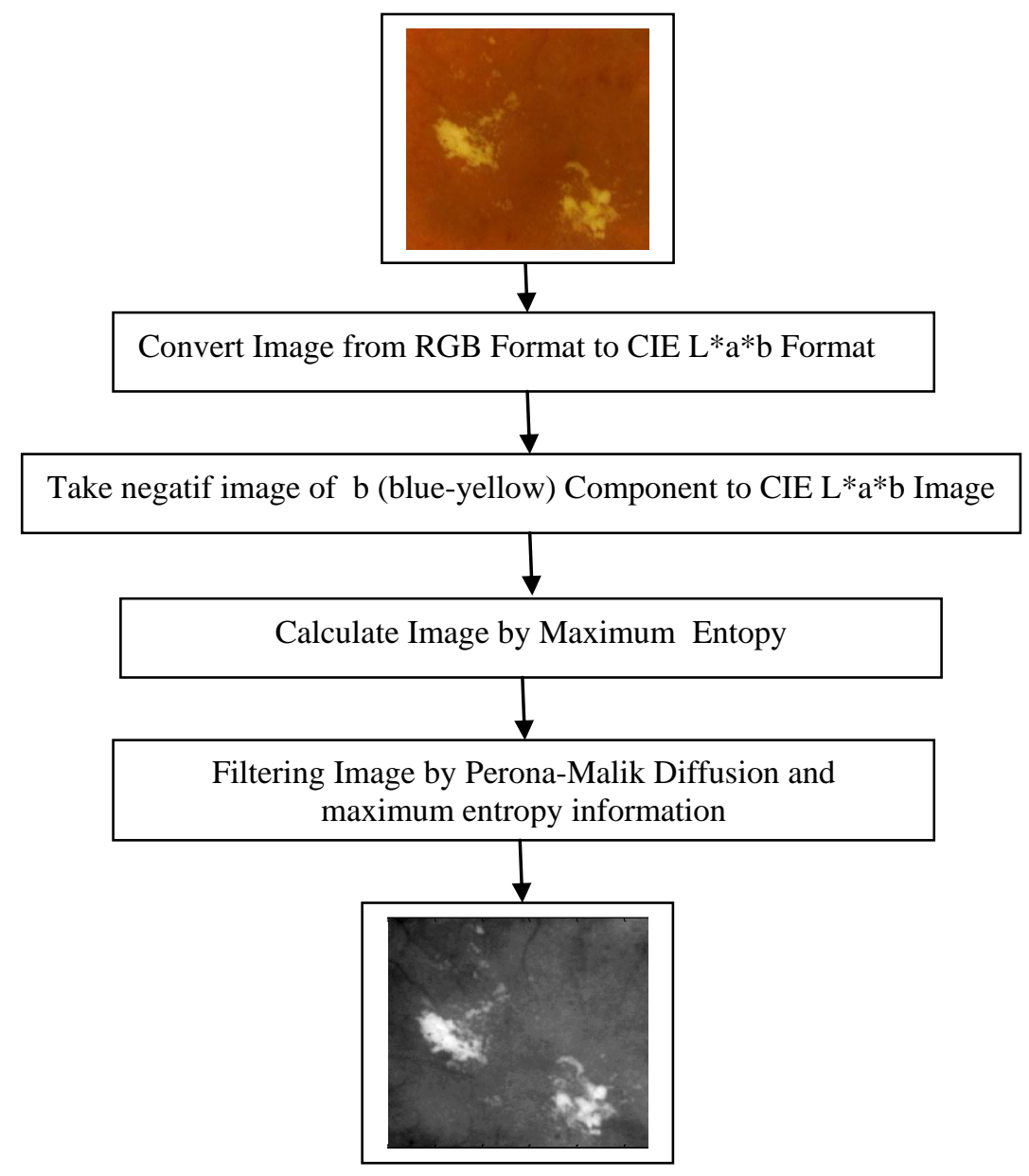

Fig. 3. Flowchart of the proposed method

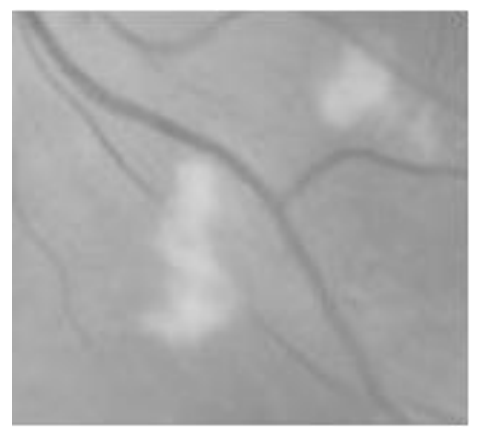

(a)

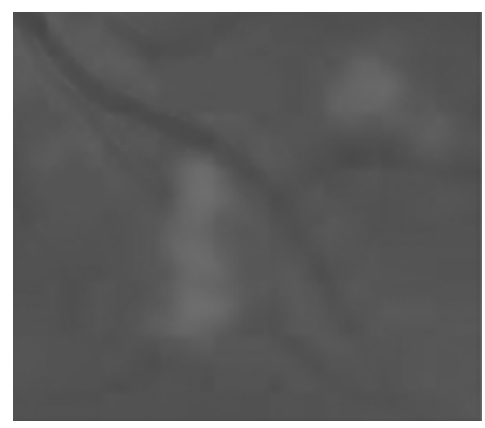

(b)

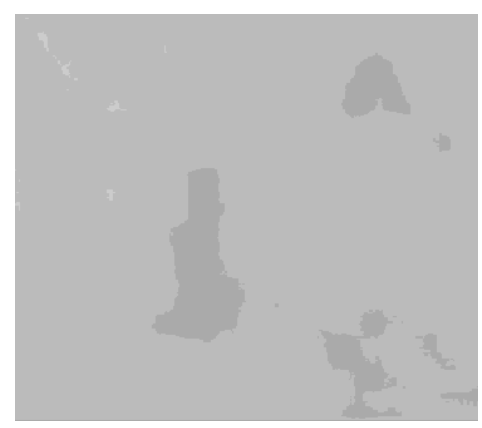

(c)

Fig. 4. (a) a component's of CIE L*a*b Image in Fig, 2 (c). (b) b component's of CIE L*a*b Image in Fig, 2 (c). (c) L component's of CIE L*a*b Image in Fig, 2 (c).

\subsection{Convert Image from RGB Format to CIE $L * a * b$ Format and Take Negatif Image of $b$ Component of CIE $L * a * b$ Image}

Fundus image has RGB format. RGB format (red, green, blue). Since some fundus images don't have uniform illumination, the Fundus image in RGB format is poorly used for the exudate segmentation process. Therefore, before it is segmented, the fundus image should be removed the illumination component of the fundus image. 
The image format which permitts to remove the illumination of image is CIE L*a*b. The CIE L* a* b image format has three components: L (illumination), a (green-red) and b (blue-yellow) elements. Therefore, in this study fundus image needs to be converted from RGB format to CIE format L*a*b. Fig. 4 (a), (b), (c) show each component of the fundus image in Fig. 2(c) in CIE L*a*b format . Component a (red-green) can be seen in Fig. 4 (a), the yellow-blue component b can be seen in Fig. 4 (b) of the CIE format L*a*b and the illumination component L can be seen in Fig. 4 (c). The experimental results in Fig. 4 show that the red-green and the blueyellow components of the CIE L*a* $\mathrm{b}$ format can distinguish the exudate on the fundus image and other areas. However, that the red-green of CIE L*a*b image in Fig. 4(a) contains retina vessel. It is very difficult to remove the retina vessel for detecting exudates in segmentation process. For this reason, we don't use red-green component, but we use the blue-yellow components of the CIE L * a *b. However, this component doesn't have exudates visualization. For this reason, this paper proposes ehanncing of the blue-yellow components of the CIE L*a *b image.

\subsection{Calculate $t$ Threshold and Enhance by Perona-Malik Diffusion}

The next step is that the $t$ threshold is calculated by maximum entropy algorithm in Section 2.2. After that, we use binary image which is calculated by using $t$ threshold information which is obtained by maximimum entropy algoritm. Therefore, the gradient of binary image is calculated for calculating speed function of PeronaMalik diffusion filter. The next stage, the Perona-Malik diffusion filter is executed to enhance the blue-yellow of CIE L*a*b component.

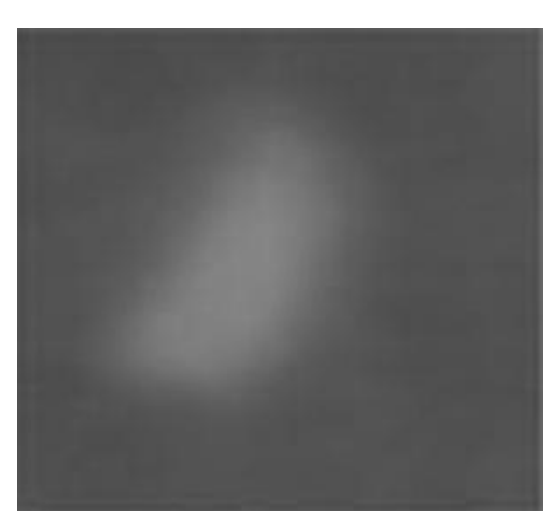

(a)

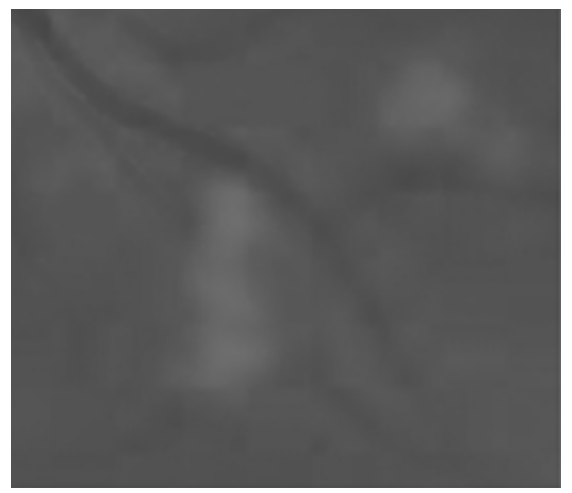

(c)

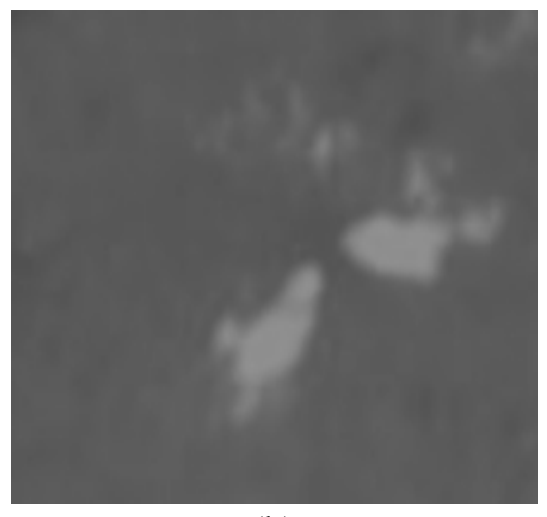

(b)

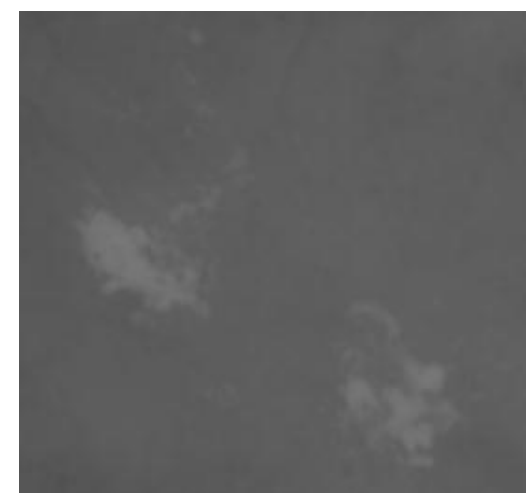

(d)

Fig. 5. (a) Negatif image of b component's of CIE L*a*b Image in Fig. 2 (a). (b) Negatif image of b component's of CIE L*a*b Image in Fig. 2 (b). (c) Negatif image of b component's of CIE L*a*b Image in Fig. 2 (c). (d) Negatif image of b component's of CIE L*a*b Image in Fig. 2 (d). 


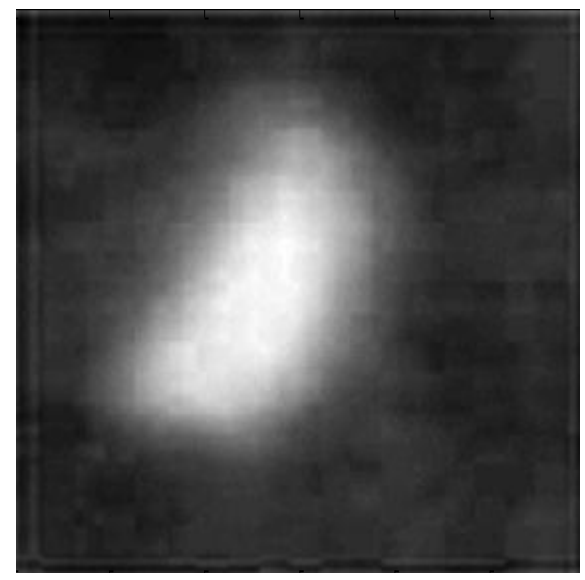

(a)

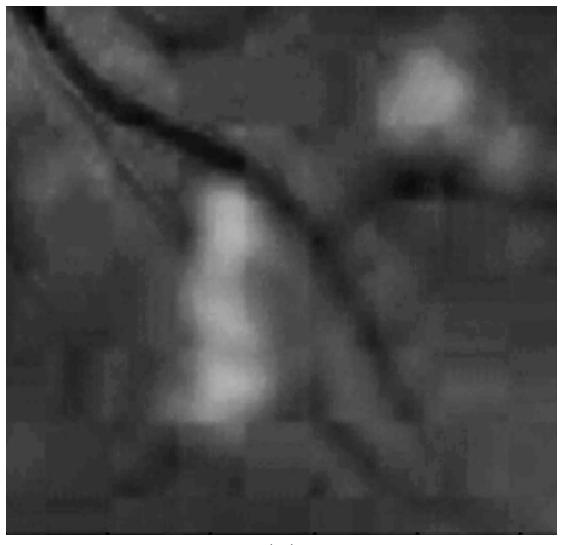

(c)

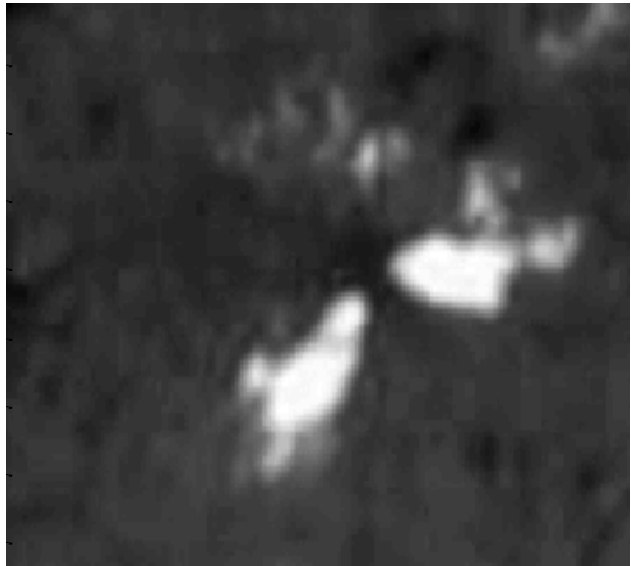

(b)

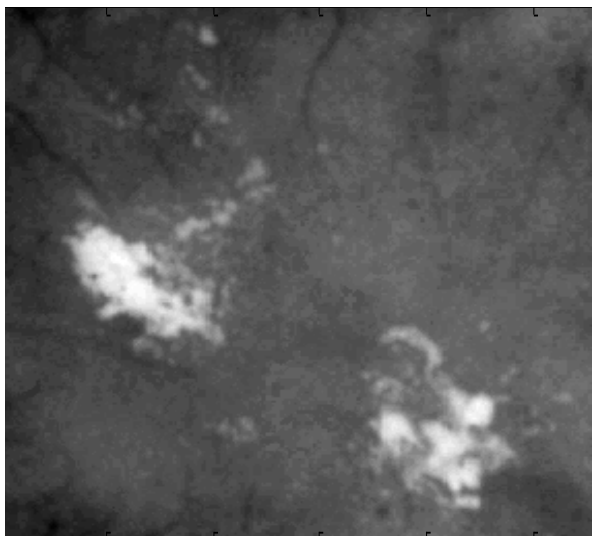

(d)

Fig. 6. (a) Enhancing Image of Fig. 2 (a) by the Perona-Malik diffusion filter with $t$ threshold information of maximum entropy. (b) Enhancing Image of Fig. 2 (b) by the Perona-Malik diffusion filter with $t$ threshold information of maximum entropy. (c) Enhancing Image of Fig 2 (c) by the Perona-Malik diffusion filter with $t$ threshold information of maximum entropy. (d) Enhancing Image of Fig 2 (d) by the Perona-Malik diffusion filter with $t$ threshold information of maximum entropy.

From the experiment results in Fig. 6, the Perona-Malik diffusion filter by using the $t$ threshold information which is decided by maximum entropy algorithm can enhance the exudates visualization in fundus image for all test images. We can compare the image before enhancing in Fig. 5 and after enhancing in Fig. 6. The exudate areas have bright areas and other areas have dark areas.

\section{Conclusion}

From the experiment results, it can be concluded that the proposed method can enhance a exudate visualization in fundus image for all test images. The illumination in the fundus image can be removed by taking $\mathrm{b}$ component of CIE L*a*b format. The Perona-Malik diffusion filter with $t$ threshold information of maximum entropy is used successfully to reduce noise in the fundus image and to enhance the exudate visualization .

\section{Acknowledgements}

We would like to say our greatest thanks to the Directorate of Research and Community Service, the Ministry of Research, Technology and Higher Education, Republic of Indonesia who has funded this research through the "Penelitian Unggulan Perguruan Tinggi (PUPT)" in 2017.

\section{References}

[1] F. Semeraro, A. Cancarini, R. Dell'Omo, S. Rezzola, M. R. Romano, and C. Costagliola, C., "Diabetic retinopathy: vascular and inflammatory disease," Journal of Diabetes Research, vol. 2015, pp.1-16, 2015. 
https://doi.org/10.1155/2015/582060

[2] J. M. Tarr, K. Kaul, M. Chopra, E. M. Kohner, and R. Chibber, "Pathophysiology of diabetic retinopathy," ISRN Ophthalmology, vol 2013, pp.1-13, 2013.

https://doi.org/10.1155/2013/343560

[3] W. Gao and J. Zuo, "Automated detection of hard exudates in fundus images using improved otsu thresholding and SVM,” International Journal of Computer Science \& Engineering Survey, vol.7, no.1, pp. 1-11, 2016. https://doi.org/10.5121/ijcses.2016.7101

[4] M. J. Cree, D. J. Cornforth, and H. F. Jelinek, "Vessel segmentation and tracking using a two-dimensional model," in Proc. of the Image and Vision, Computing Conference, 2005, pp.345-350.

[5] R. Klein, B. E. Klein, S. E. Moss, T. Y. Wong, L. Hubbard, K. J. Cruickshanks, and M. Palta, "The relation of retinal vessel caliber to the incidence and progression of diabetic retinopathy: XIX: the Wisconsin Epidemiologic Study of Diabetic Retinopathy," Archives of Ophthalmology, vol. 122, pp. 76-83, 2004.

https://doi.org/10.1001/archopht.122.1.76

[6] J. C. Russ, “The image processing handbook," 5th ed. Academic Press, New York, 2006. https://doi.org/10.1201/9780203881095

[7] P. Soille, "Morphology image analysis: principles and applications," Springer-Verlag, Telos, 1999.

[8] C. Tomasi and R. Manduchi, "Bilateral filter for gray and color images," in Proc.of the 6th international Conference on Computer Vision, pp. 839 - 846, 1998.

[9] P. Perona and J. Malik, "Scale-space and edge detection using anisotropic diffusion," IEEE Transactions on Pattern Analysis and Machine Intelligence, vol.12, pp.629 - 639, 1990.

https://doi.org/10.1109/34.56205

[10] S.Anam, E. Uchino, and N. Suetake, "Hybrid boundary detection method for image with application to coronary plaque," International Journal of Digital Information and Wireless Communications, vol.4, no.4, pp.428-437, 2014. https://doi.org/10.17781/P001297

[11] S. Anam, E Uchino, and N. Suetake, "Coronary plaque boundary enhancement in ivus image by using a modified perona-malik diffusion filter," International Journal of Biomedical Imaging, vol.2014, pp.1-9, 2014. https://doi.org/10.1155/2014/740627

[12] M. A. El-Sayed, “A new algorithm based entropic threshold for edge detection in images," International Journal of Computer Science Issues, vol. 8, issue 5, no 1, pp. 71-78, 2011. 